\title{
Space and place matter: Orte der Forschung und Entwicklung in Wien und Möglichkeiten der Stadtpolitik, sie zu unterstützen
}

Die Wahl des Standorts im Bereich Forschung und Entwicklung in Wien erfolgt manchmal aufgrund bewusster Entscheidungen und manchmal zufällig. Oft ist es gerade eine Mischung aus beidem, die zum Erfolg führt, wie Maria WIRTH in ihrer Darstellung der Entwicklung des Campus Vienna Biocenter in St. Marx zeigt ${ }^{1}$.

Es ist im Interesse der Stadt, die Ansiedelung von F\&E zu unterstützen. Es ist aber nicht gleichgültig, ob die Entscheidung über die Wahl des Standorts bewusst oder zufällig getroffen wird. Ein gewisser Grad an Steuerung unter Berücksichtigung übergeordneter raumplanerischer Prozesse und Strategien erscheint aus Sicht der Stadt sinnvoll.

Voraussetzung dafür ist die Kenntnis darüber, wo sich die Einrichtungen der unterschiedlichen F\&E-Branchen bisher niedergelassen haben - und warum. Besonders dort, wo die Konzentration einer F\&E-Branche besonders hoch ist, sind Rückschlüsse auf die Motive für die Standortwahl möglich.

Die vorliegende Studie stellt umfassend dar, wo in Wien Forschung und Entwicklung stattfindet. Neu ist dabei die Analyse der räumlichen Konzentration nach unterschiedlichen F\&E-Bereichen. Die Autoren erörtern insbesondere die Bedeutung der räumlichen Nähe und beschreiben die Motive der Betroffenen, die in Interviews erhoben wurden, als ein Erklärungsmuster für unterschiedlich hohe räumliche Konzentration in ausgewählten F\&E-Bereichen. „Local Buzz in der Wiener Forschung“ leistet einen wichtigen Beitrag zum Verständnis von Forschungsnetzwerken in der Stadt und ihrer räumlichen Verortung. Die Studie ist damit eine Grundlage für die Stadtpolitik über die Flächenwidmung und Raumplanung hinaus.

Es wird nun darum gehen, aus den neu gewonnenen Erkenntnissen konkrete Empfehlungen abzuleiten und diese zu kommunizieren, insbesondere auch innerhalb des Magistrats. So wäre beispielsweise die Bereitstellung von Raum (und Vorhalteflächen) für die Informations- und Kommunikationstechnologie rund um die Technische Universität im vierten und eventuell sechsten Bezirk eine Maßnahme der Stadt, die diesen sehr dynamischen und gleichzeitig räumlich besonders stark konzentrierten Bereich wirksam unterstützen würde.

Univ.-Prof. Dr. Alexander VAN DER BELLEN

Beauftragter der Stadt Wien für Universitäten und Forschung

\footnotetext{
1 Maria WIRTH: Der Campus Vienna Biocenter. Entstehung, Entwicklung und Bedeutung für den Life-Sciences-Standort Wien; Wien 2013
} 
